\title{
Maximum production point tracking method for a solar-boosted biogas energy generation system
}

\author{
Jialin Chen ${ }^{1}$, Ruijiang Yang ${ }^{1}$, Da Xu ${ }^{2 *}$, Bin Zhou ${ }^{1 *}$ and Yifang Jin ${ }^{1}$
}

\begin{abstract}
Low biogas yield in cold climates has brought great challenges in terms of the flexibility and resilience of biogas energy systems. This paper proposes a maximum production point tracking method for a solar-boosted biogas generation system to enhance the biogas production rate in extreme climates. In the proposed method, a multidimensional $\mathrm{R}-\mathrm{C}$ thermal circuit model is formulated to analyze the digesting thermodynamic effect of the anaerobic digester with solar energy injection, while a hydrodynamic model is formulated to express the fluid dynamic interaction between the hot-water circulation flow and solar energy injection. This comprehensive dynamic model can provide an essential basis for controlling the solar energy for digester heating to optimize anaerobic fermentation and biogas production efficiency in extreme climates. A model predictive control method is developed to accurately track the maximum biogas production rate in varying ambient climate conditions. Comparative results demonstrate that the proposed methodology can effectively control the fermentation temperature and biogas yield in extreme climates, and confirm its capability to enhance the flexibility and resilience of the solar-boosted biogas generation system.
\end{abstract}

Keywords: Renewable energy, Dynamic model, Maximum production point tracking, Model predictive control

\section{Introduction}

\subsection{Motivation}

Environmental pollution and global warming have prompted the research community to search for renewable energy sources (RESs) as valuable solutions [1]. Large-scale utilization of RESs to replace fossil energy for power generation has become the common goal of countries around the world. Biomass can play a vital role in promoting the use of RESs and is considered as the fourth largest energy resource in the world. Biomass can be converted to biogas through anaerobic fermentation, which can be used to supply local multi-energy demands using a combined heating and power (CHP) unit [2].

\footnotetext{
*Correspondence: xuda@cug.edu.cn; binzhou@hnu.edu.cn

${ }^{1}$ College of Electrical and Information Engineering, Hunan University, Changsha 410082, China

2 School of Automation, China University of Geosciences, Wuhan 430074 China
}

For mesophilic digestion, fermentation temperature is the main factor that affects the anaerobic fermentation to promote the degradation and metabolism of anaerobic bacteria [3]. Low ambient temperature can result in extremely low digestion efficiency, and the anaerobic fermentation process is suspended when the temperature falls below around zero [4]. This low-efficiency biogas production is the main obstacle to the promotion of biogas application and development in cold areas [5].

Recent studies have looked at the biogas yield problem in extreme climates. Many physical and chemical approaches have been adopted [6-9] to improve the fermentation temperature, such as straw and plastic film laying on the periphery of biogas digester, electrothermal film heating, boiler heating, waste heat heating from CHP, etc. The traditional heating approaches rely on the non-renewable fossil fuels, which release greenhouse gas during the utilization processes and have negative impact 
on the social-economic environment [10]. With the soaring price of fossil fuel and increasing environmental concerns, solar energy technologies are widely harnessed to improve anaerobic fermentation efficiency, including solar collectors and solar greenhouses [11-18]. In addition to electrical generation, CHP waste heat can be used to warm up the biogas digester, which can provide a low-cost and efficient solution for biogas production in cold areas. The inherent intermittency and volatility of solar radiation can lead to uncertainty of the external heating energy injection, thereby accelerating fermentation temperature fluctuation. Solar energy heating can be controlled by the hot-water flow to maintain the optimal temperature to ensure maximum biogas production. Therefore, this paper is devoted to establishing a solarboosted biogas generation system to manage the fermentation temperature in variable climates and rapidly track the maximum biogas production point for flexibility and resilience enhancement.

Maximum production point tracking (MPPT) involves not only the thermodynamic effects of solar energy injection but also the hydrodynamic interaction between the water flow and pipe pressure. In such cases, the fermentation temperature requires comprehensive dynamic modelling of water flow, pressure fluctuation, convective heat transfer, and conductive heat transfer in the digester climate. This could lead to high nonlinearity and increase the computational burden throughout the MPPT optimization scheme. Therefore, MPPT is a challenging problem that is difficult to solve with conventional methods because of the uncertainty of solar energy, multi-energy coupling, high-dimensional variables, nonlinear differential thermodynamics and hydrodynamics.

\subsection{Literature review}

Traditional physical insulation measures, such as covering the ground around the digester with charcoal [9], laying straw and plastic film on the periphery of the biogas digester, are commonly used to prevent heat loss and maintain fermentation temperature. However, by adding insulation materials, the fermentation temperature can only be passively sustained rather than proactively adjusted. Thus, several studies have looked at solving the low biogas yield problem with external energy injection. In [4], engine waste heat is employed to provide the steam required for thermal hydrolysis pre-treatment and the heat for anaerobic digestion. Electricity from natural gas and fossil fuel to heat the anaerobic digestion is also effective in improving the biogas yield [7], though waste of primary energy and negative impact on the environment cannot be avoided.

The focus of recent research is to use RESs to facilitate relatively high fermentation temperature, including the ground source heat pump [19], biomass boiler [20], groundwater source heat pump [6], methane liquid heat recovery pump etc. However, considering availability and economy, these methods are not suitable for biogas production in rural areas. Compared with other renewable technologies, solar energy is independent of the influence of the geographic environment and offers eco-friendly and least expensive heating sources [4, 21]. It has been proved that solar heating is an efficient way to facilitate biogas production. In [15], a solar heating biogas fermentation system is tested for five months and the heat collected by the $2 \mathrm{~m}^{2}$ solar collector after eight hours in October can meet the heat demands of $6 \mathrm{~m}^{3}$ biogas digester's fermentation at Hohhot. A solar heated biogas system integrated with a phase change thermal storage device is introduced in [16] and the preliminary results show that the system could be a promising solution to promote the application of biogas technology in cold areas. The solar-heated biogas digester is modeled in [17] to simulate and predict its thermal behavior. Two solar greenhouses are used to heat the digester in [18] and the simulation indicates that the proposed system can increase biogas production and revenue compared to the system with no heating resource. Nevertheless, existing work has mainly focused on the enhancement of steadystate production performance while trying to verify the economical and technical feasibilities of the solar-heated biogas system. The system thermodynamic and hydrodynamic behaviors, which are essential for the development of advanced control strategies to track the maximum biogas production, are ignored.

\subsection{Contribution and organization}

In this paper, a model predictive control (MPC)-based biogas MPPT method is proposed to enhance the biogas production rate of the solar-boosted biogas generation system in extreme climates. The contributions of the paper are:

1. A dynamic model of a solar-boosted biogas energy generation system is formulated to describe the thermodynamic and hydrodynamic interactions during the biogas production process with solar energy injection. An R-C thermal network is modeled to analyze the digesting thermodynamic effect with solar energy injection, while a hydrodynamic model is formulated to control the solar hot-water circulation flow. Compared with the previous solar-heated biogas system with uncontrollable dynamic modelling, this model allows accurate control of thermodynamic and hydrodynamic behaviors, which can provide an essential basis for controlling the solar energy for digester heating. 
2. A MPC-based biogas MPPT method is proposed to maximize the biogas production rate in variable climates. The strategy systematically integrates the rolling optimization and feedback mechanism based on short-term prediction of the solar radiation and ambient temperature, to optimize the digesting fermentation temperature, so as to track the maximum biogas production rate and enhance the resilience of the solar-boosted biogas generation system to varying ambient climate conditions.

The rest of the paper is organized as follows. Section 2 illustrates the low biogas yield problem in extreme climates and shows the proposed biogas generation system. Section 3 describes the proposed MPC-based MPPT method, while Sect. 4 analyzes the performance of the proposed method through simulation. Section 5 presents the conclusions.

\section{Dynamic modelling of solar-boosted biogas generation system}

\subsection{Problem description}

Among all the factors that affect the anaerobic fermentation, temperature is of greatest importance. For mesophilic anaerobic fermentation, the increase in temperature of the anaerobic digester will promote the degradation and metabolism of anaerobic bacteria, thus improving the biogas production [22]. Therefore, solar energy is utilized for biogas digester heating to enhance the fermentation temperature in this paper. The hotwater circulation for biogas digester heating is illustrated in Fig. 1. As can be seen, the cold water in the tank is pumped to the solar thermal collector. Then solar radiation is absorbed and converted to thermal energy for water heating in the pipe, which is transmitted to the digester tank to heat the biogas fermentation liquid. However, with further increase of fermentation temperature, such overtemperature can reduce the activity of

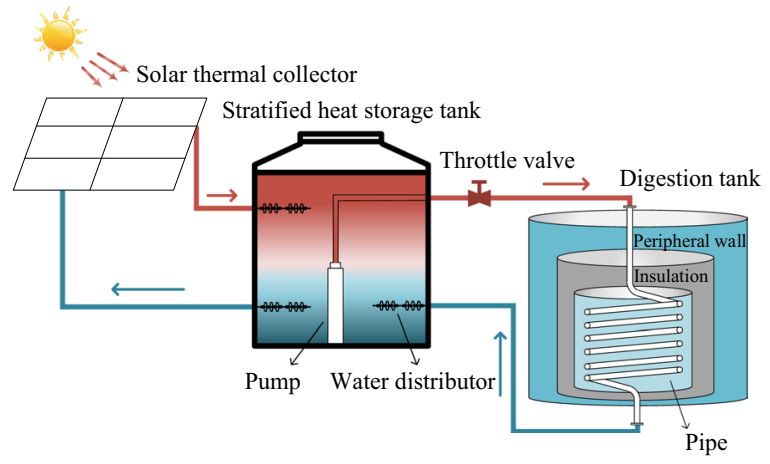

Fig. 1 Solar hot-water circulation for biogas digester heating microorganisms in the fermentation process, resulting in inefficient biogas production. Therefore, it is important to develop control strategies that can track the maximum biogas production by optimizing the fermentation temperature.

This paper aims to maintain the fermentation temperature at the optimal level so that the system can output maximum production in real time, i.e., to achieve MPPT. With the digesting thermodynamic effects on biogas production, the fermentation temperature can be influenced by external solar energy injection. The heat transmitted to the digester can be controlled by the hot-water flow, while water flow is highly nonlinear to the pressure drop along pipelines based on hydrodynamic analysis. In addition, the inherent intermittency and volatility of solar radiation make the analysis of the associated optimal control problem challenging. In order to maintain optimal biogas production, the primary objectives of this paper are to propose: a biogas generation system based on thermodynamic and hydrodynamic analysis, and a MPC-based MPPT to enable the biogas generation system to rapidly track the desired maximum point during dynamic changes of ambient temperature.

\subsection{Modelling of solar-boosted biogas generation system}

\section{Digesting thermodynamic analysis}

For biogas digestion, temperature is the primary environmental factor that affects the digestion rate and process. In general, the increase of fermentation temperature will lead to the increase of the substrate use and microbial metabolic rate, thereby improving the biogas production. However, if the fermentation temperature exceeds the optimum temperature, the activity of anaerobic bacteria and the efficiency of the digestion process will be reduced. According to the field measured data in [23], biogas production rate $f_{b i o}$ is a function of fermentation temperature and can be modeled as:

$$
f_{\text {bio }}=\frac{B_{\mathrm{o}} S_{\mathrm{o}}}{H R T}\left(1-\frac{K}{H R T \mu_{\mathrm{m}}-1+K}\right)
$$

where $B_{o}$ is the biochemical methane potential; $S_{o}$ is the influent volatile solids concentration; $H R T$ is the hydraulic retention time; $\mu_{m}$ is the maximum specific growth rate of microorganisms; $T_{d}$ is the fermentation temperature [24]. $K$ is a dimensionless kinetic parameter related to the concentration of influent volatile solids [25], and is given as:

$$
K=0.6+0.0206 \mathrm{e}^{0.051 S_{\mathrm{o}}}
$$


The relationship between the maximum specific growth rate of microorganisms and the fermentation temperature is given as [25]:

$$
\mu_{\mathrm{m}}=\left\{\begin{array}{l}
0.026 \mathrm{e}^{0.1319 T_{\mathrm{d}}}, 0^{\circ} \mathrm{C} \leq T_{\mathrm{d}} \leq 30^{\circ} \mathrm{C} \\
0.013 T_{\mathrm{d}}-0.129,30^{\circ} \mathrm{C} \leq T_{\mathrm{d}} \leq 60^{\circ} \mathrm{C}
\end{array}\right.
$$

The available solar energy can be used for digester heating, thereby promoting the anaerobic fermentation process for biogas production enhancement [26]. A multi-dimensional R-C thermal circuit model of the underground biogas digester is established to analyze the digesting thermodynamic effects under external energy injection. Here, the heat transfer and storage are represented by thermal resistances and capacitances. The inside, internal wall, peripheral wall and ambient temperatures of the digestion reactor can be characterized by nodes with thermal potentials. Using the lumped model analysis of the structure and environmental conditions of the cylindrical digester in [24], three thermal circuital branches are established to analyze the nodal digesting thermodynamic effects on different surfaces. For the $i_{t \mathrm{~h}}$ branch, the internal wall is the insulation layer and can be denoted as a node connected to the node inside the digester by two resistances $Z_{\text {ini } i} / 2$ and $Z_{\mathrm{d} i}$, and the peripheral wall node is connected to the external node by two series resistances $Z_{\mathrm{w} i} / 2$ and $Z_{\text {outi }}$. Two resistances $Z_{\mathrm{in} i} / 2$ and $Z_{\mathrm{w} i} / 2$ are used to represent the thermal interaction and transfer of the two-layer walls. Thus, the digesting thermodynamic effects can be modeled based on the nodal analysis of thermal circuits, as:

$$
\begin{gathered}
C_{\mathrm{d}} \frac{d T_{\mathrm{d}, k}}{d k}=E_{\mathrm{sf}, k}+\frac{T_{\mathrm{in} 1, k}-T_{\mathrm{d}, k}}{Z_{\mathrm{d} 1}+\frac{Z_{\mathrm{in} 1}}{2}}+\frac{T_{\mathrm{in} 2, k}-T_{\mathrm{d}, k}}{Z_{\mathrm{d} 2}+\frac{Z_{\mathrm{in} 2}}{2}} \\
\quad+\frac{T_{i n 3, k}-T_{d, k}}{Z_{d 3}+\frac{Z_{\text {in } 3}}{2}} \\
C_{i n i} \frac{d T_{\mathrm{in} i, k}}{d k}=\frac{T_{\mathrm{d}, k}-T_{\mathrm{in} i, k}}{Z_{\mathrm{d} i}+Z_{\mathrm{in} i} / 2}+\frac{T_{w i, k}-T_{\mathrm{in} i, k}}{Z_{\mathrm{w} i} / 2+Z_{\mathrm{in} i} / 2}, \forall i=1,2,3 \\
C_{w 1} \frac{d T_{w 1, k}}{d k}=\frac{T_{o u t, k}-T_{w 1, k}}{Z_{\text {out }}+Z_{w 1} / 2}+\frac{T_{i n 1, k}-T_{w 1, k}}{Z_{i n 1} / 2+Z_{w 1} / 2} \\
C_{w 2} \frac{d T_{w 2, k}}{d k}=\frac{T_{\mathrm{s}, k}-T_{w 2, k}}{Z_{\mathrm{s}}+Z_{w 2} / 2}+\frac{T_{i n 2, k}-T_{w 2, k}}{Z_{i n 2} / 2+Z_{w 2} / 2} \\
C_{w 3} \frac{d T_{w 3, k}}{d k}=\frac{T_{\mathrm{s}, k}-T_{w 3, k}}{Z_{\mathrm{s}}+Z_{w 3} / 2}+\frac{T_{i n 3, k}-T_{w 3, k}}{Z_{i n 3} / 2+Z_{w 3} / 2} \\
E_{s f, k}=C_{\mathrm{water}, k} Q_{\mathrm{d}, k} \rho_{\mathrm{water}}\left(T_{\mathrm{hw}, k}-T_{d, k+1}\right)
\end{gathered}
$$

where $C_{\mathrm{d}}, C_{\mathrm{in} i}$, and $C_{\mathrm{w} i}$ represent the thermal capacitances of the digester inside, the $i_{\text {th }}$ surface of the insulation layer and the peripheral wall, respectively; $Z_{\mathrm{d} i}, Z_{\text {out }}$, $Z_{\mathrm{in} i}, Z_{\mathrm{w} i}$ and $Z_{\mathrm{s}}$ represent the thermal resistances that are used for the convective heat transfer inside the digester and through the digester cover, conductive heat transfer inside, peripheral walls and soil, respectively; $T_{\mathrm{d}}, T_{\text {out }}$, $T_{\mathrm{s}}, T_{\mathrm{ini} i}$, and $T_{\mathrm{w} i}$ are the temperatures inside and outside the digester, the surrounding soil, the first and second layer walls, respectively; $k$ represents the operating time; $\rho_{\text {water }}$ is the water density; $E_{\text {sf }}$ represents the controllable amount of thermal energy injection from renewable harvesting; $T_{\mathrm{hw}, k}$ is the temperature of hot-water flow injected into the digester at time $k$.

\section{Hydrodynamic modelling of solar water circulation}

The heat transmitted to the digester can be controlled by the hot-water flow. A hydrodynamic networking model is established to describe the interaction between the water flow and the pressure drop along the pipelines. Solar collector, heat storage tank, throttle valve and biogas fermentation tank can be represented by nodes with specific pressure as shown in Fig. 2. Between adjacent nodes, the flow coefficient of the pipeline is used to describe the flow resistance, and there is a nonlinear relationship between water flow and pressure drop along the transmission pipeline [27]. According to the node analysis of the hydrodynamic network loop, the water flow $Q_{\mathrm{d}, k}$ from the throttle valve to the digester will change with the pressure at the throttle valve outlet, which can be described as:

$$
\begin{aligned}
& Q_{\mathrm{d}, k}=\alpha_{\mathrm{bp} 4} \sqrt{U_{\mathrm{val}, \text { out }, k}-U_{\mathrm{d}, k}} \\
& 0 \leq U_{\mathrm{d}, k} \leq U_{\mathrm{val}, \text { out }, k}
\end{aligned}
$$

where $\alpha_{\mathrm{bp}}$ is the flow coefficient of the pipeline; $U_{\mathrm{d}, k}$ is the pressure of the biogas digester at time $k ; U_{\mathrm{val}, \mathrm{out}, k}$ is the flow pressure at the outlet.

Other water flows as well as their temperatures in the model are described as:

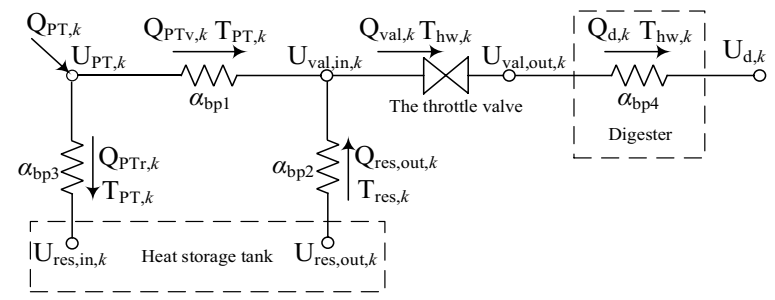

Fig. 2 Hydrodynamic model of the biogas generation system 


$$
\begin{aligned}
& Q_{\mathrm{PTV}, k}=\alpha_{\mathrm{bp} 1} \sqrt{U_{\mathrm{PT}, k}-U_{\mathrm{val}, \mathrm{in}, k}} \\
& Q_{\mathrm{res}, \text { out }, k}=\alpha_{\mathrm{bp} 2} \sqrt{U_{\mathrm{res}, \text { out }, k}-U_{\mathrm{val}, \mathrm{in}, k}} \\
& Q_{\mathrm{PTr}, k}=\alpha_{\mathrm{bp} 3} \sqrt{U_{\mathrm{PT}, k}-U_{\text {res,in }, k}} \\
& T_{\mathrm{hw}, k}=\frac{Q_{\mathrm{PTv}, k} T_{\mathrm{PT}, k}+Q_{\mathrm{res}, \text { out }, k} T_{\mathrm{res}, k}}{Q_{\mathrm{PTv}, k}+Q_{\text {res }, \text { out }, k}} \\
& Q_{\mathrm{PT}, k}=Q_{\mathrm{PTv}, k}+Q_{\mathrm{PTr}, k}
\end{aligned}
$$

where $Q_{\text {res,out }, k}$ is the water flow from the heat storage tank to the throttle valve; $Q_{\mathrm{PTv}, k}$ is the total water flow from the collector; $Q_{\mathrm{PTV}, k}, Q_{\mathrm{PTr}, k}$ are the water flows from the collector to the throttle valve and the tank, respectively; $U_{\text {res,in, } k}$ and $U_{\text {res,out }, k}$ are the respective inlet and outlet pressures of the tank; $U_{\mathrm{PT}, k}$ and $U_{\mathrm{val}, \mathrm{in}, k}$ are the respective pressures of the solar collector and inlet of the throttle valve; $T_{\mathrm{PT}, k}$ and $T_{\text {res }, k}$ are the temperatures of the collector and tank at time $k$, respectively.

The throttle valve controls the water flow by changing the throttling area. Assuming that the throttling process is an isenthalpic process, the water flow through the throttle valve at time $k Q_{\mathrm{val}, k}$ has the following relationship with the inlet and outlet pressures:

$$
\begin{aligned}
& Q_{v a l, k}=\beta_{\text {val }} A_{\text {val }, k} \sqrt{\frac{2\left(U_{\mathrm{val}, i n, k}-U_{\mathrm{val}, o u t, k}\right)}{\rho_{\text {water }}}} \\
& Q_{\text {val }, k}=Q_{\mathrm{PTv}, k}+Q_{\text {res }, \text { out }, k} \\
& A_{v a l, \text { min }} \leq A_{\text {val.k }} \leq A_{\text {val,max }} \\
& U_{v a l, i n, \min } \leq U_{\mathrm{val}, i n, k} \leq U_{v a l, i n, \max } \\
& U_{\text {val,out, } \min } \leq U_{\mathrm{val}, \text { out }, k} \leq U_{\text {val,out,max }}
\end{aligned}
$$

where $\beta_{\mathrm{val}}$ is the flow coefficient; $A_{\mathrm{val}, k}$ is the throttling area at time $k ; U_{\mathrm{val}, \mathrm{in}, k}$ is the flow pressure at the inlet; $A_{\text {val,min }}$ and $A_{\text {val,max }}$ are the respective lower and upper limits of the allowable throttle area; $U_{\mathrm{val}, \text { in,min }}, U_{\mathrm{val}, \text { in,max }}$, $U_{\mathrm{val}, \text { out,min }}$ and $U_{\mathrm{val}, \text { out,max }}$ are the allowable pressure limits at the inlet and outlet of the throttle valve. The throttling area of the valve can be changed by adjusting the opening of the valve, so as to control the water flow. It is noted from (17) that the change of throttling area will lead to a pressure change at the outlet of the throttle valve, as:

$$
U_{\mathrm{val}, \text { out }, k}=U_{\mathrm{val}, i n, k}-\frac{Q_{v a l, k}^{2} \rho_{\mathrm{water}}}{2 \beta_{\mathrm{val}}^{2} A_{\text {val }, k}^{2}}
$$

When the light and heat are insufficient, the heat storage tank provides heat so the water flow of the throttle valve is equal to the outlet water flow of the storage tank and the collector. In contrast, when the light and heat are sufficient, the excess thermal energy is stored in the tank so the water flow of the throttle valve is equal to the water flow of the collector.

3. Solar-biogas generation system for multi-energy supplies

The use of solar energy to heat the biogas digester is efficient in overcoming the obstacle of low biogas yield in a cold climate and improving solar energy penetration. Figure 3 illustrates a solar-biogas generation system with multi-energy carriers, where solar and biomass energy are converted into electricity and heat through the solar thermal collector and biogas digester. Given the thermodynamic effects of fermentation temperature on biogas production, the thermal energy from the solar collector is used to heat the biogas digester, thereby promoting anaerobic fermentation to enhance the biogas production rate. The biogas can be stored in the biogas storage tank or converted into electricity and heat via a CHP unit to supply local multi-energy demands [28]. The hot-water storage tank and biogas storage tank can provide sufficient storage capacity for the available heat and biogas. Therefore, a variety of energy conversion and storage devices are integrated to combine and couple these carriers to form a centerinternal energy redundant connection, and can provide flexibility and synergy for multi-energy supplies.

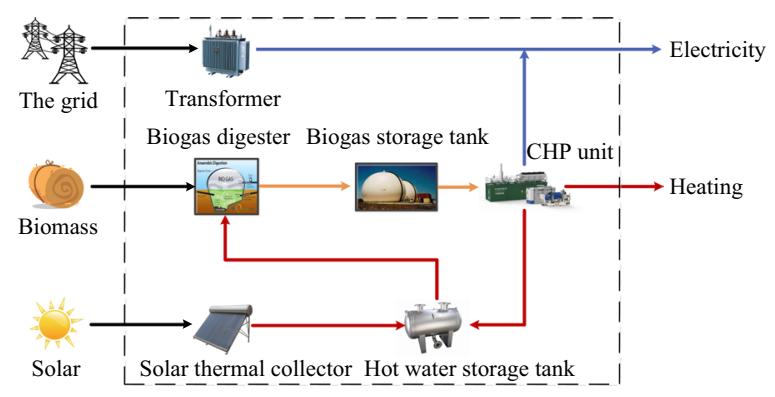

Fig. 3 Solar-boosted biogas generation system for multi-energy supplies 


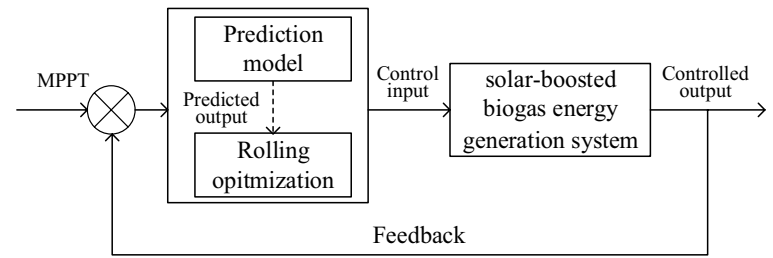

Fig. 4 Block diagram of MPC-based MPPT

\section{MPC-based MPPT method}

\subsection{MPPT framework based on MPC}

Figure 4 shows the MPC-based MPPT framework. The predicted solar radiation and ambient temperature can be obtained from the historical observation data using a nonlinear regression method [3]. Monte Carlo simulations are implemented to generate a series of scenarios to capture the prediction uncertainties of solar radiation and ambient temperature [5]. Thus the fermentation temperature and biogas production rate in each time slot can be obtained from the multi-dimensional $\mathrm{R}-\mathrm{C}$ thermodynamic model of the biogas digester and the hydrodynamic model of the hot-water circulation flow based on the prediction scenarios and input parameters.

During the optimization process, the control decision is obtained by solving the optimization problem at the current time while taking into account the uncertainties of solar radiation and ambient temperature of future horizons [29], while only the optimal control decision for the current time $k$ is implemented as the actual control input [30].

Considering the inaccuracy of the predicted outputs obtained from the proposed model, a feedback mechanism is adopted based on the actual outputs of the solarboosted biogas generation system at the current time $k$ to tune the multi-dimensional $\mathrm{R}-\mathrm{C}$ thermodynamic model of the biogas digester and the hydrodynamic model of the hot-water circulation flow, as well as the prediction of solar radiation and ambient temperature.

\subsection{Objective function}

The optimization objective aims to minimize the difference between the maximum biogas production and the biogas production at the current time $\mathrm{k}$, as well as the expected biogas production of all remaining future scenarios, which can be formulated as:

$$
\begin{aligned}
& \min \left\{\left(f_{\mathrm{bio}, \text { max }}-f_{\mathrm{bio}, k, s}^{*}\right)^{2}+\sum_{t=k+\Delta k}^{K_{\text {end }}} \sum_{s=1}^{N_{s}} \pi_{s}\left(f_{\mathrm{bio}, \text { max }}-f_{\mathrm{bio}, t, s}^{*}\right)^{2}\right\} \\
& T_{\mathrm{d}, \text { min }} \leq T_{\mathrm{d}, k}^{*} \leq T_{\mathrm{d}, \max }
\end{aligned}
$$

where $K_{\text {end }}$ represents the last time slot of the rolling horizon; $N_{\mathrm{s}}$ represents the total number of scenarios; $\pi_{\mathrm{s}}$ is the probability of scenario $s ; f_{\text {bio, } k, s}$ is the calculated biogas production at time $k$; and $f_{\text {bio,max }}$ is the maximum biogas production of the mesophilic fermentation digestion.

\subsection{System constraints}

\section{Solar thermal collector}

The flat-plate solar collectors have simple mechanical structure and stable performance, and are widely used in household and industrial applications. They usually include a black absorber with a glass cover and insulation. While the glass cover with low iron content can increase light transmittance and reduce radiation loss, the insulation on the back and sides can reduce conduction losses. The plate can absorb and convert solar radiation into useable heat to heat the water up to $90{ }^{\circ} \mathrm{C}$ while the thermal efficiency can reach $70 \%$. The useful energy $E_{\mathrm{PT}, k}$ is calculated based on the energy balance as [31]:

$$
\begin{aligned}
& E_{P T, k}=G_{P T, k} A_{\mathrm{s}} \eta_{\mathrm{s}}=C_{\mathrm{water}, k} Q_{\mathrm{PT}, k} \rho_{\mathrm{water}}\left(T_{\mathrm{PT}, k}-T_{\mathrm{out}, k}\right) \\
& 0 \leq T_{\mathrm{PT}, k} \leq T_{\mathrm{PT}, \max } \\
& 0 \leq Q_{\mathrm{PT}, k} \Delta k \leq V_{\mathrm{PT}, \max } \\
& U_{\mathrm{PT}, \min } \leq U_{\mathrm{PT}, k} \leq U_{\mathrm{PT}, \max }
\end{aligned}
$$

where $G_{\mathrm{PT}, k}$ is the solar radiation at time $k ; A_{\mathrm{S}}$ is the lighting area of the collector; $\eta_{\mathrm{s}}$ is the efficiency of the solar collector; $C_{\text {water }}$ is the specific heat capacity of the hotwater in the solar thermal collector; $V_{\mathrm{PT}, \max }$ is the rated volume of the collector; $U_{\mathrm{PT}, \min }$ and $U_{\mathrm{PT}, \max }$ are the allowable pressure limits of the collector.

\section{Stratified heat storage tank}

Solar energy has significant instability and discontinuity due to the changes of weather and other factors. Storage of solar energy is necessary when the fermentation temperature exceeds the optimal temperature, while the hot-water stored in the tank when the solar radiation intensity is sufficient can provide thermal feedback for the biogas digester in an underlit scene, such as a cloudy day or night.

A natural layered heat storage tank is adopted, and its specific structure is shown in Fig. 1. The tank is directly connected to the solar thermal collector where the outlet and return pipes are located above and below the tank, respectively. The inlet ports of the solar collector and the outlet ports of the biogas tank are located at the high temperature top of the tank whereas the outlet ports of 
the solar collector and the inlet ports of the biogas tank are located at the low temperature bottom of the tank. The density of water varies with temperature, so the returning water tends to flow up or down to the storage tank because its temperature is significantly different from the temperature at the top and bottom of the tank [32].

Assuming that the heat losses in and out of the water pipe are negligible, the energy balance of the heat storage tank is given as:

$$
\begin{aligned}
& E_{\text {res }, \text { total }, k+1}=E_{\text {res }, \text { total }, k}+E_{\text {res }, i n, k}-E_{\text {res }, \text { out }, k}-E_{\text {res,loss }, k} \\
& =E_{\text {res }, \text { total }, k}+m_{\text {res }, k} C_{\text {water }}\left(T_{\text {res }, k+1}-T_{\text {res }, k}\right. \\
& E_{\text {res }, \text { loss }, k}=\theta_{\mathrm{hl}} A_{\text {res }}\left(T_{\text {res }, k}-T_{\text {out }, k}\right) \\
& E_{\text {res }, i n, k}=C_{\text {water }} Q_{\mathrm{PTr}, k} \rho_{\text {water }}\left(T_{\mathrm{PT}, k}-T_{\text {res }, k+1}\right) \\
& E_{\text {res }, \text { out }, k}=C_{\text {water }} Q_{\text {res }, \text { out }, k} \rho_{\text {water }}\left(T_{\text {res }, k}-T_{\mathrm{d}, k+1}\right)
\end{aligned}
$$

The operating constraints of the tank are:

$$
\begin{aligned}
& m_{\text {res, } k+1}=m_{\text {res }, k}+\left(Q_{\mathrm{PTr}, k}-Q_{\text {res,out }, k}\right) \rho_{\text {water }} \Delta k \\
& E_{\text {res, }, \text { total,min }} \leq E_{\text {res,total }, k} \leq E_{\text {res,total,max }} \\
& U_{\text {res,out,min }} \leq U_{\text {res,out }, k} \leq U_{\text {res,out,max }} \\
& U_{\text {res,in,min }} \leq U_{\text {res,in, }, \mathrm{k}} \leq U_{\text {res,in,max }} \\
& U_{\text {res,out,min }} \leq U_{\text {res,out }, k} \leq U_{\text {res,out,max }} \\
& V_{\text {res, } \text { min }} \rho_{\text {water }} \leq m_{\text {res, }, k} \leq V_{\text {res, } \text { max }} \rho_{\text {water }}
\end{aligned}
$$

where $E_{\text {res,total }, k}$ and $E_{\text {res,loss, } k}$ are the total heat and heat loss in the tank at time $k$, respectively; $m_{\text {res, } k}$ is the mass of the hot-water in the tank at time $k ; A_{\text {res }}$ is the surface area of the tank; $E_{\mathrm{res}, \text { in }, k}$ and $E_{\mathrm{res}, \mathrm{out}, k}$ are the inlet and outlet heat in the tank at time $k$, respectively; $T_{\text {out }, k}$ is the temperature of the outside of the tank at time $k ; \theta_{\mathrm{hl}}$ is the heat loss coefficient; $U_{\text {res,in,min }}, U_{\text {res,in,max }}, U_{\text {res,out,min }}$ and $U_{\text {res.out,max }}$ are the allowable pressure limits at the inlet and outlet of the tank; $V_{\text {res,min }}$ and $V_{\text {res,max }}$ are the lower and upper limits of the hot-water volume, respectively.

\subsection{Solution method}

\section{Problem reformulation}

MPPT is a nonlinear programming optimization problem which is difficult to solve directly using existing solvers because of the nonlinear differential thermodynamics and hydrodynamics. In such cases, the thermodynamics-based R-C thermal network and the hydrodynamic networking model are reformulated to decrease the nonlinearity and computational burden.

As the multi-dimensional $\mathrm{R}-\mathrm{C}$ thermal circuit model in (4)-(9) is highly nonlinear, the linearization method of the general nonlinear differential system in [33] is adopted to decrease the nonlinearity of the digesting thermodynamic equations. It has been demonstrated in [33] that this linearization will not result in noticeable error. This is because of the small fermentation temperature range of the biogas digester. Thus, the linearized state space form of the multi-dimensional $\mathrm{R}-\mathrm{C}$ thermal model is discretized as:

$$
\mathrm{T}_{k+1, i}=\mathrm{AT}_{k, i}+\mathrm{Bu}_{k}
$$

where $\mathbf{T}_{k, i}=\left[T_{\mathrm{in}, k, i}, T_{\mathrm{w}, k, i}, T_{\mathrm{s}, k, i}\right]$ is the state vector representing the nodal temperatures of the $\mathrm{R}-\mathrm{C}$ thermal model at time $k ; \mathbf{u}_{k}$ denotes the input vector including solar energy injection and environmental temperature; $\mathbf{A}$ and $\mathbf{B}$ are the coefficient matrices which can be obtained from the capacitances and resistances of thermal circuital branches.

In order to decrease the nonlinearity of water flows in (10)-(14), a linearization method in [5] is applied to linearize the hydrodynamic model of hot-water circulation flow. In Fig. 5, $Q_{\mathrm{d}, k}$ is selected as an example to illustrate the linearization process. As seen, point $\mathrm{O}\left(W_{4, k}^{0}, Q_{\mathrm{d}, k}^{0}\right)$ represents the base point, around which the hot-water flow equation is linearized, while point $\mathrm{A}\left(W_{4, k}, Q_{\mathrm{d}, k}\right)$ and point $\mathrm{B}\left(W_{4, k}^{\mathrm{L}}, Q_{\mathrm{d}, k}\right)$ represent the current operating point and linear prediction point, respectively. $W_{4, k}$ denotes the squared pressure drop. $W_{4, k}$ and $Q_{\mathrm{d}, k}$ are given as:

$$
\begin{aligned}
& W_{4, k}=U_{\mathrm{d}, k}-U_{\mathrm{val}, \text { out }, k} \\
& Q_{\mathrm{d}, k}=\alpha_{\mathrm{bp}} \sqrt{W_{4, k}}
\end{aligned}
$$

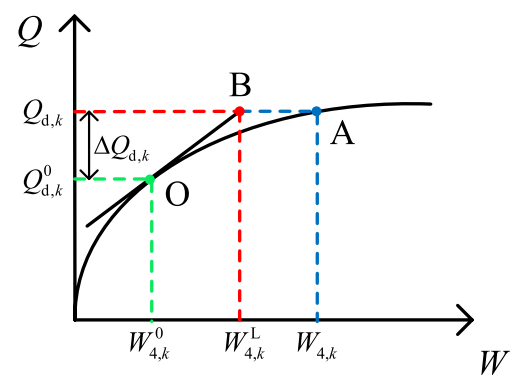

Fig. 5 Linearization of hydrodynamic model 
$W_{4, k}^{\mathrm{L}}$ can be obtained from the linearized water flow equations around point $\mathrm{O}$, as:

$$
\begin{gathered}
W_{4, k}^{\mathrm{L}}=W_{4, k}^{0}+\Delta Q_{\mathrm{d}, k} /\left.\frac{d Q_{\mathrm{d}, k}}{d W_{4, k}}\right|_{0} \\
\left.\frac{d Q_{\mathrm{d}, k}}{d W_{4, k}}\right|_{0}=\frac{\alpha_{4}}{2 \sqrt{W_{4, k}^{0}}}=\frac{\alpha_{4}^{2}}{2 Q_{\mathrm{d}, k}^{0}}
\end{gathered}
$$

In addition, the capacitance and resistance parameters of the dynamic models can be derived from [12, 22, 27], and have been validated against the historical data (e.g., digestion temperature, outside temperature, thermal energy for digester heating, etc.) for parameter tuning [3].

\section{Solution procedures}

Figure 6 illustrates the flowchart of the proposed MPPT method procedure. In this paper, an MPPT method is proposed to improve the fermentation temperature and biogas yield in extreme climates considering the thermodynamic and hydrodynamic effects, thereby enhancing the flexibility and resilience of the solar-boosted biogas generation system. Specifically, for the multi-dimensional $\mathrm{R}-\mathrm{C}$ thermal circuit model, the fermentation temperature is determined by all heat changes inside the digester, including the hot water injected into the digester, outside temperature, etc. For the hydrodynamic networking model, the solar water circulation is determined by all pressure changes inside the digester, including the pressure at the throttle valve outlet, heating water from renewables, etc. Based on the reformulated thermodynamics-based $\mathrm{R}-\mathrm{C}$ thermal network and the hydrodynamic networking model in Sect. 3.4, the MPC-based MPPT is adopted. The optimization problem is solved every $15 \mathrm{~min}$ in a $24-\mathrm{h}$ rolling horizon and only the first control step is implemented at the current time $k$. Considering the inaccuracy of the parameters and prediction data, a feedback mechanism is executed to adjust the control decisions of the next time slot. All the tests are performed via the commercial Matlab platform on a laptop with 1.8-GHz Intel Core i7 CPU and 8 GB RAM, and solved with Yalmip solver CPLEX with their default settings. The optimized results of $24 \mathrm{~h}$ can be obtained and further analyzed to calculated the system performance index for comparative study.

\section{Case study}

\subsection{Experimental data and settings}

In this case study, the proposed MPPT method is tested on a demonstration project in Yiyang, Hunan, China.

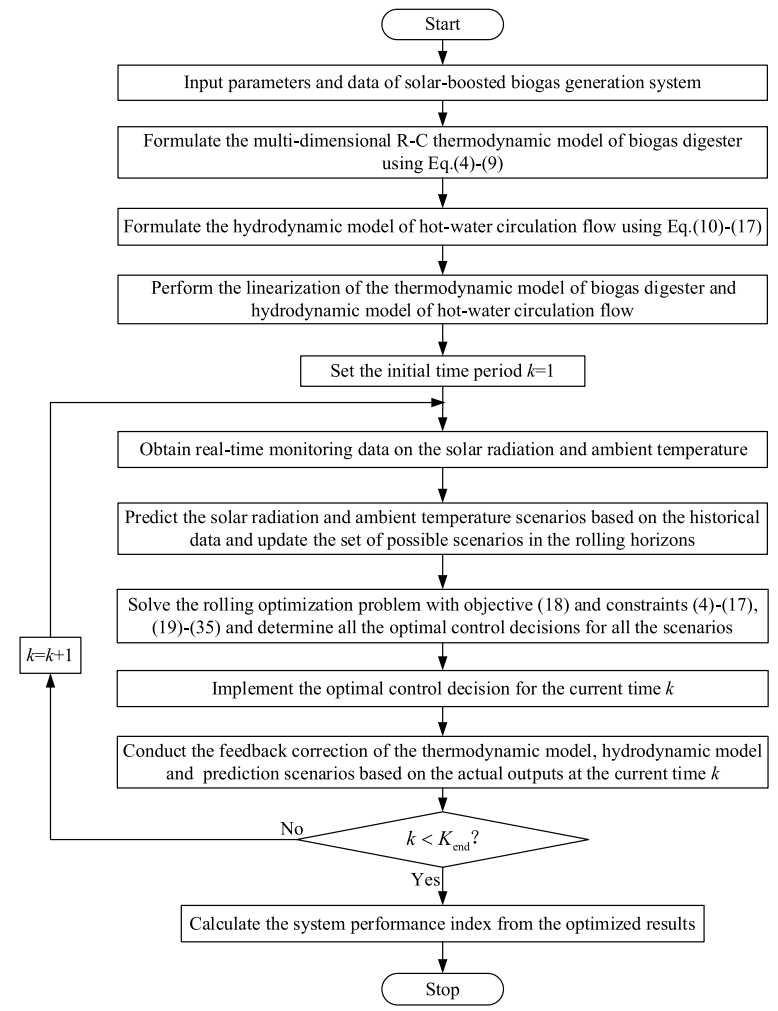

Fig. 6 Flowchart of the MPPT method solution procedure

The project is equipped with a $600 \mathrm{~m}^{3}$ cylindrical biogas digester and a $600 \mathrm{~m}^{3}$ gas tank for biogas production and storage. The actual parameters of the biogas digester, solar thermal collector, and stratified heat storage tank from the rural microgrid demonstration are shown in Table 1.

The complete scheduling cycle $\mathrm{k}$ and time interval $\Delta \mathrm{k}$ of the biogas generation system are set to $24 \mathrm{~h}$ and $1 \mathrm{~h}$ respectively. Monte Carlo simulations are performed to generate a series of scenarios to capture the prediction uncertainties of solar radiation and ambient temperature. The initial scenario tree consists of 2000 scenarios, and each scenario has a $1 / 2000$ probability of occurrence. In order to decrease the computational effort, only 10 scenarios are reserved for rolling optimization in each time slot, as shown in Fig. 7. Because of the seasonal differences in meteorological conditions, typical daily meteorological data in summer and winter in Hunan are adopted, as shown in Figs. 8 and 9.

\subsection{Comparative results and analysis}

In order to validate the effectiveness and superior performance of the proposed methodology, comparative studies are conducted with three schemes: 
Table 1 Technical specifications of the solar-boosted biogas generation system $[5,23]$

\begin{tabular}{|c|c|c|}
\hline Device & Power factors & Power generation efficiency \\
\hline \multirow[t]{9}{*}{ Digester } & $Z_{d}=0.062 K / W$ & $Z_{\text {in } 1}=0.58 \mathrm{~K} / \mathrm{W}$ \\
\hline & $Z_{\text {in } 2}=0.66 \mathrm{~K} / \mathrm{W}$ & $Z_{\text {in } 3}=0.61 \mathrm{~K} / \mathrm{W}$ \\
\hline & $Z_{\mathrm{w} 1}=0.21 \mathrm{~K} / \mathrm{W}$ & $Z_{\mathrm{w} 2}=0.29 \mathrm{~K} / \mathrm{W}$ \\
\hline & $Z_{\mathrm{w} 3}=0.25 \mathrm{~K} / \mathrm{W}$ & $C_{d}=0.46 \mathrm{kWh} / \mathrm{K}$ \\
\hline & $C_{s 1}=10.21 \mathrm{kWh} / \mathrm{K}$ & $C_{s 2}=10.92 \mathrm{kWh} / \mathrm{K}$ \\
\hline & $C_{53}=10.18 \mathrm{kWh} / \mathrm{K}$ & $C_{\mathrm{w} 1}=5.26 \mathrm{kWh} / \mathrm{K}$ \\
\hline & $C_{\mathrm{w} 2}=5.87 \mathrm{kWh} / \mathrm{K}$ & $C_{\mathrm{W} 3}=5.09 \mathrm{kWh} / \mathrm{K}$ \\
\hline & $H R T=25$ days & $S_{0}=100 \mathrm{~kg} / \mathrm{m}^{3}$ \\
\hline & $T_{\mathrm{d}, \min }=0^{\circ} \mathrm{C}$ & $T_{\mathrm{d}, \max }=60^{\circ} \mathrm{C}$ \\
\hline \multirow{3}{*}{$\begin{array}{l}\text { Solar thermal } \\
\text { collector }\end{array}$} & $A_{\mathrm{s}}=150 \mathrm{~m}^{2}$ & $\eta_{s}=0.65$ \\
\hline & $T_{\mathrm{PT}, \max }=90^{\circ} \mathrm{C}$ & $V_{P T, \text { max }}=300 \mathrm{~L}$ \\
\hline & \multicolumn{2}{|c|}{$C_{\text {water }}=0.0012 \mathrm{kWh} /\left(\mathrm{kg}^{\circ} \mathrm{C}\right)$} \\
\hline \multirow{2}{*}{$\begin{array}{l}\text { Stratified heat } \\
\text { storage tank }\end{array}$} & $E_{\text {res,total, } \min }=0$ & $E_{\text {res,total,max }}=80 \mathrm{kWh}$ \\
\hline & $V_{\text {res, } \min }=0 \mathrm{~L}$ & $V_{\text {res, } \max }=3000 \mathrm{~L}$ \\
\hline
\end{tabular}

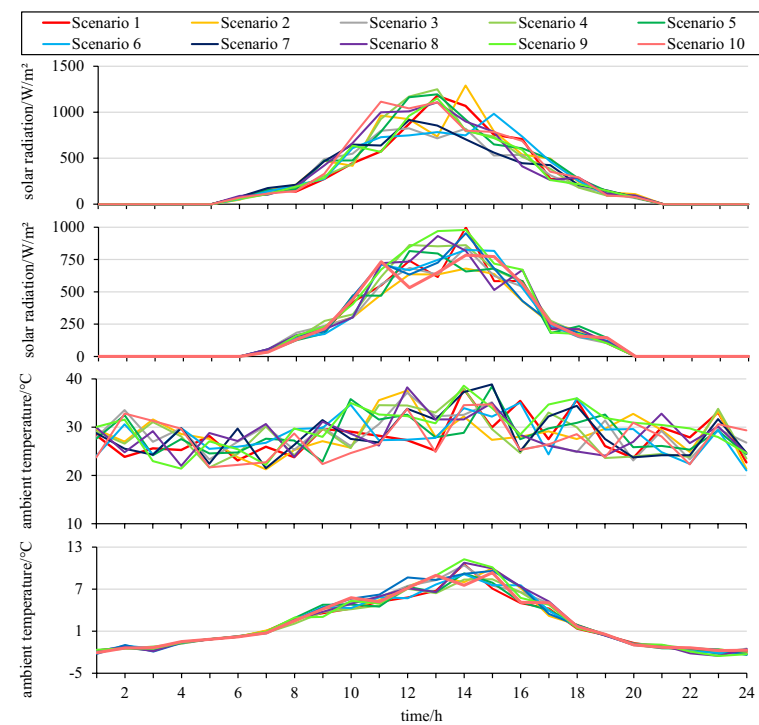

Fig. 7 The daily solar radiation and ambient temperature of 10 scenarios

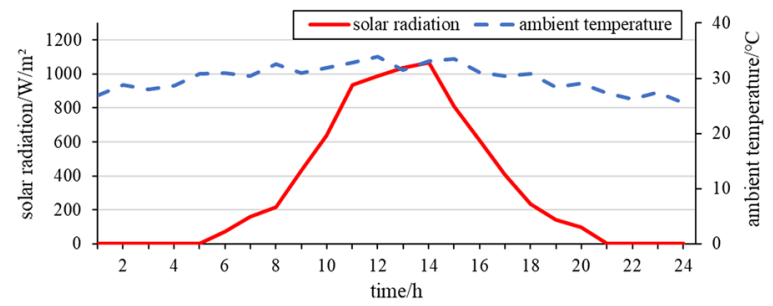

Fig. 8 Typical average daily solar radiation and ambient temperature in summer
1. Scheme 1 is the proposed MPC-based MPPT method in Sects. 2 and 3.

2. Scheme 2 is the solar-boosted biogas generation system without adopting the MPPT method.

3. Scheme 3 is the biogas generation system without using the solar energy.

Figures 10 and 11 depict the dynamic changes in water temperature and the volume of heated water inside the solar thermal collector in summer and winter, respectively. As can be seen, the solar collector stops working during the nighttime because of zero solar radiation, and thus the water temperature of the collector is equal to the environmental temperature and the volume of heated water is 0 . During the daytime, the collector absorbs solar radiation so both the water temperature and the volume of heated water increase. It can be seen that the heated water volume follows the temperature, such as during $6 \mathrm{~h}$ to $14 \mathrm{~h}$ in winter, for full absorption of solar radiation

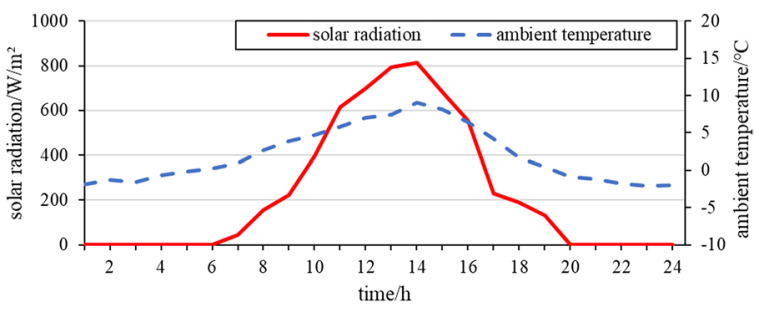

Fig. 9 Typical average daily solar radiation and ambient temperature in winter

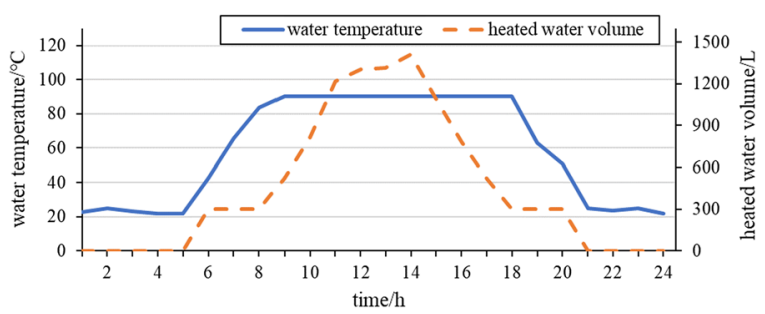

Fig. 10 Water temperature and heated water volume of collector in summer

under the protection temperature limit.

The dynamic changes of heat storage/release and the remaining heat of the stratified heat storage tank in summer and winter are illustrated in Figs. 12, 13, 14 and 15. The positive and negative values in Figs. 12 and 13 represent heat storage and heat release, respectively. It should be noted that the change of heat storage in the tank is mainly affected by the ambient temperature and solar 


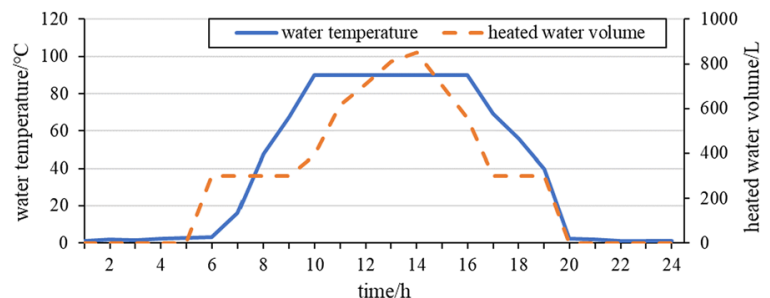

Fig. 11 Water temperature and heated water volume of collector in winter

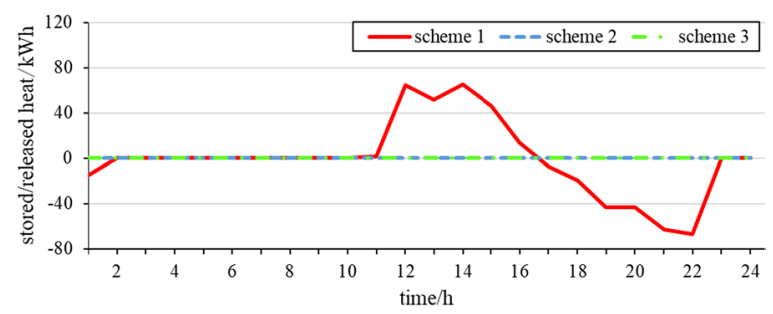

Fig. 12 Dynamic change of heat storage/release of the tank in summer

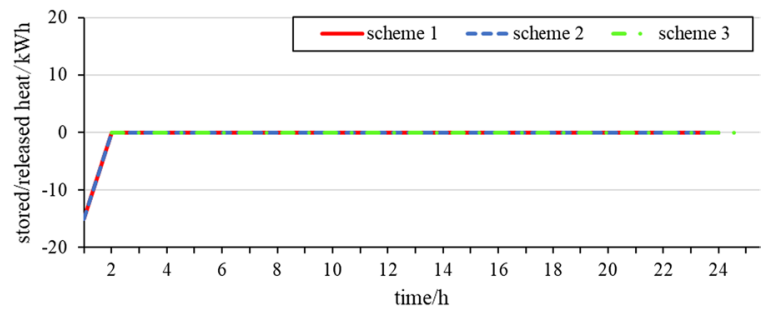

Fig. 13 Dynamic change of heat storage/release of the tank in winter

radiation. For example, the increment of heat storage at $13 \mathrm{~h}$ decreases in summer time as shown in Fig. 12 as the magnitude of the solar radiation increase is less than the temperature decrease. As the solar radiation in winter is much less than that in summer, no excess heat can be used for storage in winter. Thus, the dynamic change of the heat storage/release and the remaining heat of the storage tank in Scheme 1 are completely consistent with those in Scheme 2, as shown in Fig. 13. Furthermore, as can be seen from Figs. 12 and 14, excess thermal energy in scheme 1 is automatically stored from 12 to $16 \mathrm{~h}$ and released from 16 to $21 \mathrm{~h}$ to improve the fermentation temperature because of the nodal pressure regulation by the MPPT method. The surplus heat in scheme 2 cannot be stored in the storage tank, and thus no heat can be used for promoting the biogas yield at night, such as during $19 \mathrm{~h}$ to $23 \mathrm{~h}$ in summer.

As shown in Figs. 16 and 17, the contribution of solar energy feedback to heat the digester in schemes 1 and

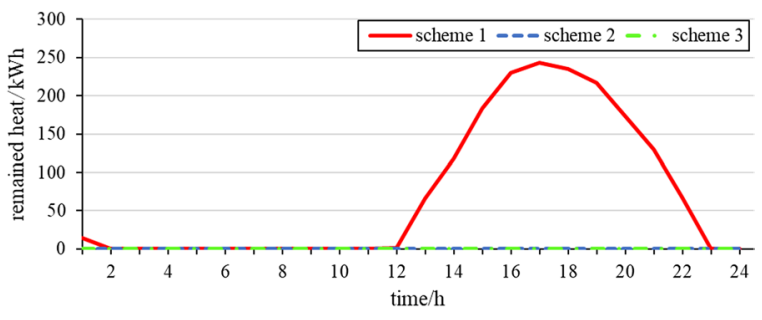

Fig. 14 Dynamic change of remaining heat of the tank in summer

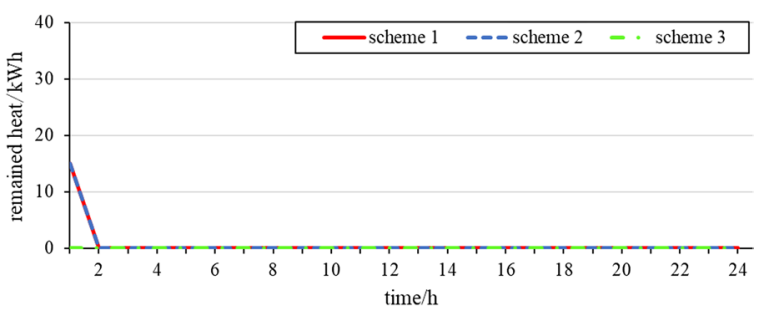

Fig. 15 Dynamic change of remaining heat of the tank in winter

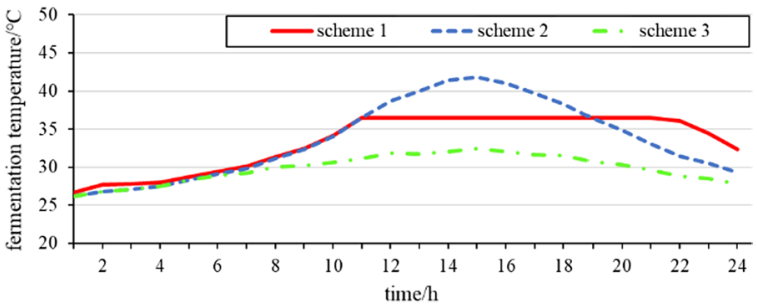

Fig. 16 Biogas fermentation temperature in summer

2 , and thus to the fermentation temperature, increases biogas production compared with scheme 3 . Since the MPPT method is not considered in scheme 2, the surplus thermal energy cannot be stored in the tank, and has to be directly injected into the digester. As a result, the fermentation temperature from 11 to $19 \mathrm{~h}$ in scheme 2 is above the optimal temperature, which leads to the decrease of biogas yield as the activity of anaerobic microorganisms is affected by the high temperature. The proposed methodology outperforms scheme 2 by maintaining the optimal fermentation temperature and biogas yield from 11 to $22 \mathrm{~h}$, with the excess solar energy fully utilized and stored in hot-water.

Figures 18 and 19 depict the curves of biogas fermentation temperature and yield in winter. The average daily fermentation temperature in winter is $5.37^{\circ} \mathrm{C}$ in both schemes 1 and 2, which is more than twice as high as that in scheme 3 . Since the fermentation temperature in scheme 3 is below $8{ }^{\circ} \mathrm{C}$ all day and below $0{ }^{\circ} \mathrm{C}$ for the first $7 \mathrm{~h}$, the anaerobic bacteria can barely survive in winter, and thus the total production of biogas in scheme 3 is 0 . It is clear that schemes 1 and 2 reduce the low-temperature 


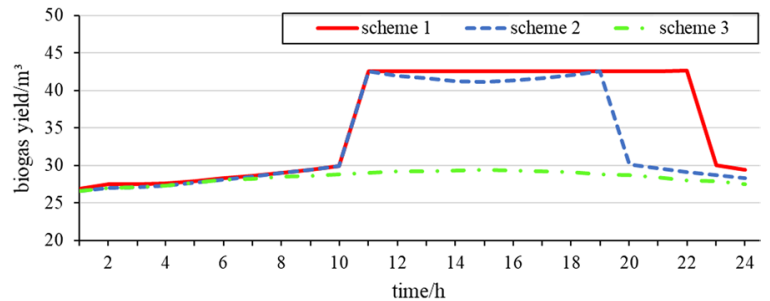

Fig. 17 Biogas yield in summer

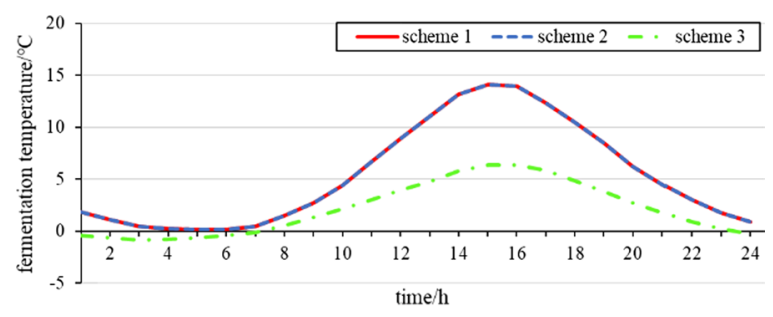

Fig. 18 Biogas fermentation temperature in winter

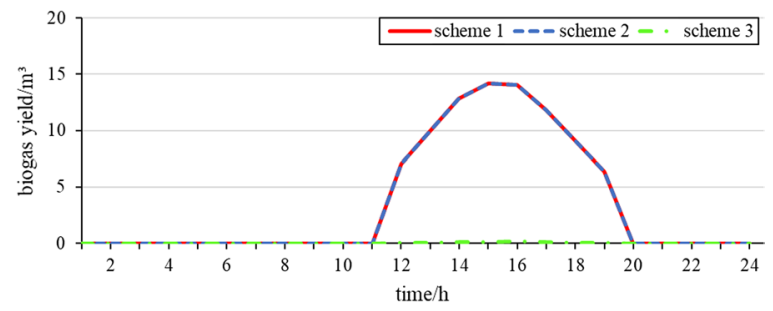

Fig. 19 Biogas yield in winter

time. This can maintain the activity of the anaerobic bacteria. Compared with scheme 3, sufficient solar thermal energy is used to maintain the optimal fermentation temperature in schemes 1 and 2, and this enhances the biogas production even in cold climates.

The 24-h comparative performance results on the average fermentation temperature, biogas yield, electricity procurement cost, gas procurement cost, and biogas supply break time with schemes $1-3$ on typical summer and winter days are summarized in Tables 2 and 3 , respectively. Considering that electricity is the main energy requirement of local consumers for the incomplete district heating network in the south of China, the CHP unit is running in the following-electric-load (FEL) mode with fixed electrical generation efficiency of 0.4 and thermal generation efficiency of 0.45 [3]. As shown in Figs. 20, 21, 22 and 23, the thermal and electrical outputs cannot completely meet the local demand in schemes $1-3$. Hence, the CHP unit has to consume all generated biogas at every moment in
Table 2 Comparative results of schemes 1-3 on typical summer days

\begin{tabular}{llll}
\hline Scheme & \multicolumn{3}{l}{ Summer } \\
\cline { 2 - 4 } & $\mathbf{1}$ & $\mathbf{2}$ & $\mathbf{3}$ \\
\hline Average fermentation temperature $\left({ }^{\circ} \mathrm{C}\right)$ & 33.56 & 33.67 & 29.80 \\
Biogas yield $\left(\mathrm{m}^{3}\right)$ & 852.63 & 802.91 & 681.43 \\
Electricity procurement cost $(Y)$ & 693.06 & 776.12 & 979.08 \\
Gas procurement cost $(Y)$ & 88.64 & 104.68 & 143.88 \\
Biogas supply break time $(\mathrm{h})$ & 0 & 0 & 0 \\
\hline
\end{tabular}

Table 3 Comparative results of schemes 1-3 on typical winter days

\begin{tabular}{llll}
\hline Scheme & \multicolumn{3}{l}{ Winter } \\
\cline { 2 - 4 } & $\mathbf{1}$ & $\mathbf{2}$ & $\mathbf{3}$ \\
\hline Average fermentation temperature $\left({ }^{\circ} \mathrm{C}\right)$ & 5.37 & 5.37 & 2.09 \\
Biogas yield $\left(\mathrm{m}^{3}\right)$ & 85.27 & 85.27 & 0 \\
Electricity procurement cost $(Y)$ & 1251.78 & 1251.78 & 1394.23 \\
Gas procurement cost $(Y)$ & 367.82 & 367.82 & 402.21 \\
Biogas supply break time $(\mathrm{h})$ & 15 & 15 & 24 \\
\hline
\end{tabular}

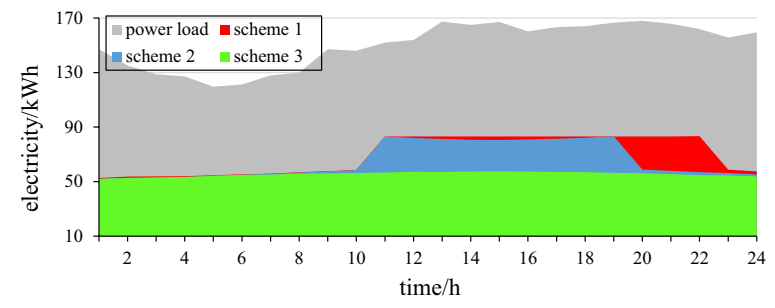

Fig. 20 Electricity energy outputs of CHP unit in summer

the FEL mode for energy supply without excess biogas reserved, and will be shut down during biogas supply break time. Furthermore, considering a better coordination among the collector and heat storage tank under the proposed MPPT method in scheme 1, it can be seen that scheme 1 in summer can reduce the procurement cost of electricity and gas by $11.25 \%$ compared with scheme 2. The biogas supply break time in schemes 1 and 2 can also be reduced by $9 \mathrm{~h}$ compared to scheme 3 in winter, because of the solar energy feedback from the collector. The comparative results demonstrate the superior performance of the proposed scheme 1 for the improvements in electricity and gas procurement cost and biogas supply break time. This confirms its capability to enhance the flexibility and resilience of the solarboosted biogas generation system. 


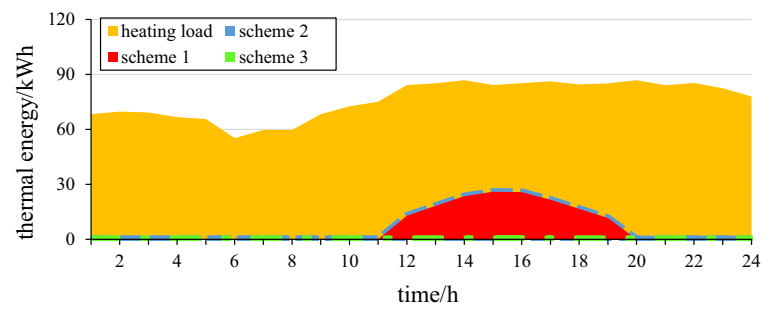

Fig. 23 Thermal energy outputs of $\mathrm{CHP}$ unit in winter

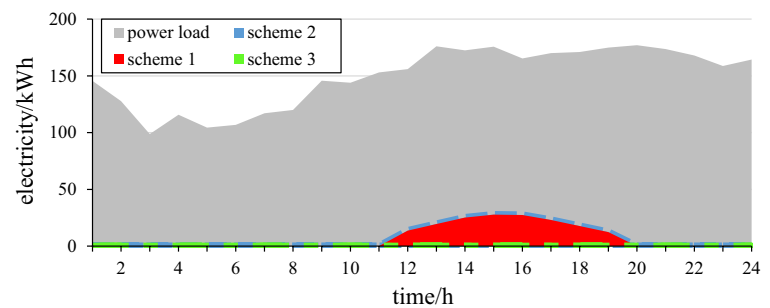

Fig. 21 Electricity energy outputs of CHP unit in winter

\section{Conclusions}

In this paper, a solar-boosted biogas energy generation system considering thermodynamic and hydrodynamic effects is established to control the solar energy for digester heating. A MPC-based MPPT method is developed to enhance the biogas production rate in extreme climates. The effectiveness and validity of the proposed methodology have been extensively tested on a demonstration project equipped with a $600 \mathrm{~m}^{3}$ cylindrical biogas digester in Hunan, China. The conclusions of this investigation are summarized as follows:

1. The proposed method can effectively enhance the flexibility and resilience of the solar-boosted biogas generation system, especially for the low biogas yield problem in extreme climates.

2. The proposed method can make full use of the collected solar energy and prevent excessive fermentation temperature in summer by storing the excess heat in the stratified heat storage tank.

3. The proposed method can maintain the optimal fermentation temperature and track the maximum biogas production in varying climate conditions.

\section{Acknowledgements}

Not applicable.

\section{Authors' contributions}

The authors contributed equally to the development of this research. All authors read and approved the final manuscript.

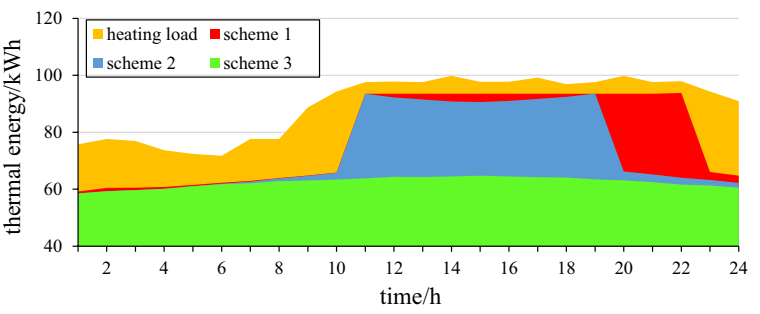

Fig. 22 Thermal energy outputs of CHP unit in summer

\section{Funding}

This work was supported in part by the National Natural Science Foundation of China under Grant 51877072, in part by the Hunan Natural Science Foundation of China under Grant 2021 JJ10019, in part by Huxiang Young Talents Programme of Hunan Province under Grant 2019RS2018, and in part by the China Postdoctoral Science Foundation funded project under Grant 2021 M692992.

Availability of data and materials

Not applicable.

\section{Declarations}

\section{Competing interests}

The authors declare that they have no known competing financial interests or personal relationships that could have appeared to influence the work reported in this paper.

Received: 16 August 2021 Accepted: 21 November 2021

Published online: 18 December 2021

\section{References}

1. Shang, L., Zhu, W., Li, P., et al. (2018). Maximum power point tracking of PV system under partial shading conditions through flower pollination algorithm. Protection and Control of Modern Power Systems, 3(1), 1-7.

2. Badal, F., Das, P., Sarker, S., et al. (2019). A survey on control issues in renewable energy integration and microgrid. Protection and Control of Modern Power Systems, 4(1), 1-27.

3. Zhou, B., Xu, D., Li, C., et al. (2018). Optimal scheduling of biogas-solarwind renewable portfolio for multicarrier energy supplies. IEEE Transactions on Power Systems, 33(6), 6229-6239.

4. Tian, G., Yang, B., Dong, M., et al. (2018). The effect of temperature on the microbial communities of peak biogas production in batch biogas reactors. Renewable Energy, 123, 15-25.

5. Zhang, K., Zhou, B., Li, C., et al. (2020). Dynamic modelling and coordinated multi-energy management for a sustainable biogas-dominated energy hub. Energy, 220, 119640

6. Su, B., Han, W., \& Jin, H. (2017). Proposal and assessment of a nove integrated CCHP system with biogas steam reforming using solar energy. Applied Energy, 206, 1-11.

7. Mirmasoumi, S., Saray, R., \& Ebrahimi, S. (2018). Evaluation of thermal pretreatment and digestion temperature rise in a biogas fueled combined cooling, heat, and power system using exergo-economic analysis. Energy Conversion and Management, 163, 219-238.

8. Zheng, Y., Wei, J., Li, J., et al. (2012). Anaerobic fermentation technology increases biomass energy use efficiency in crop residue utilization and biogas production. Renewable and Sustainable Energy Reviews, 16(7), 4588-4596

9. Yadvika, S., Sreekrishnan, T., et al. (2004). Enhancement of biogas production from solid substrates using different techniques-A review. Bioresource Technology, 95(1), 1-10.

10. Collet, P., Flottes, E., Favre, A., et al. (2017). Techno-economic and Life Cycle Assessment of methane production via biogas upgrading and power to gas technology. Applied Energy, 192, 282-295. 
11. Li, C., Yang, H., Shahidehpour, M., et al. (2020). Optimal planning of islanded integrated energy system with solar-biogas energy supply. IEEE Transactions on Sustainable Energy, 11(4), 2437-2448.

12. Feng, R., Li, J., Dong, T., et al. (2016). Performance of a novel household solar heating thermostatic biogas system. Applied Thermal Engineering, 96, 519-526.

13. Rahman, M., Hasan, M., Paatero, J., et al. (2014). Hybrid application of biogas and solar resources to fulfill household energy needs: A potentially viable option in rural areas of developing countries. Renewable Energy, 6(8), 35-45.

14. Cheng, J., Zhang, C., Sun, J., et al. (2019). Sustainability accounting for the construction and operation of a plant-scale solar-biogas heating system based on emergy analysis. International Journal of Energy Research, 43(8), 3806-3822

15. Su, Y., Tian, R., \& Yang, X. (2011). Research and analysis of solar heating biogas fermentation system. Procedia Environmental Sciences, 11, 1386-1391.

16. Lu, Y., Tian, Y., Lu, H., et al. (2015). Study of solar heated biogas fermentation system with a phase change thermal storage device. Applied Thermal Engineering, 88, 418-424.

17. Axaopoulos, P., Panagakis, P., Tsavdaris, A., et al. (2001). Simulation and experimental performance of a solar-heated anaerobic digester. Solar Energy, 70(2), 155-164.

18. Hassanein, A., Ling, Q., Pan, J., et al. (2015). Simulation and validation of a model for heating underground biogas digesters by solar energy. Ecological Engineering, 82, 336-344.

19. Gaballah, E., Abdelkader, T., Luo, S., et al. (2020). Enhancement of biogas production by integrated solar heating system: A pilot study using tubular digester. Energy, 193, 0360-5442.

20. Zhang, T., Tan, Y., \& Zhang, X. (2016). Using a hybrid heating system to increase the biogas production of household digesters in cold areas of China: An experimental study. Applied Thermal Engineering, 103, 1299-1311.

21. Hamdi, H., Regaya, C., \& Zaafouri, A. (2019). A sliding-neural network control of induction-motor-pump supplied by photovoltaic generator. Protection and Control of Modern Power Systems, 5(1), 1-17.

22. Weatherford, V., \& Zhai, Z. (2015). Affordable solar-assisted biogas digesters for cold climates: Experiment, model, verification and analysis. Applied Energy, 146, 209-216.

23. Chen, Y., \& Hashimoto, A. (1978). Kinetics of methane fermentation. Biotechnology and Bioengineering Symposium, 8(4), 503-511.

24. Liu, Y., Chen, Y., Li, T., et al. (2017). Investigation on the heat loss characteristic of underground household biogas digester using dynamic simulations and experiments. Biosystems Engineering, 163, 116-133.

25. Hashimoto, A. (1982). Methane from cattle waste: Effects of temperature, hydraulic retention time, and influent substrate concentration on kinetic parameter (k). Biotechnology and Bioengineering, 24(9), 2039-2052.

26. Xu, D., Zhou, B., Liu, N., et al. (2021). Peer-to-peer multienergy and communication resource trading for interconnected microgrids. IEEE Transactions on Industrial Informatics, 17(4), 2522-2533.

27. Fooladivanda, D., \& Taylor, J. (2018). Energy-optimal pump scheduling and water flow. IEEE Transactions on Control of Network Systems, 5(3), 1016-1026.

28. Li, X., Li, W., Zhang, R., Jiang, T., et al. (2020). Collaborative scheduling and flexibility assessment of integrated electricity and district heating systems utilizing thermal inertia of district heating network and aggregated buildings. Applied Energy, 258, 114021.

29. Yin, L., \& Su, Z. (2021). Multi-step depth model predictive control for photovoltaic power systems based on maximum power point tracking techniques. International Journal of Electrical Power and Energy Systems, 131, 107075.

30. Batiyah, S., Sharma, R., Abdelwahed, S., et al. (2020). An MPC-based power management of standalone DC microgrid with energy storage. International Journal of Electrical Power and Energy Systems, 120, 105949.

31. Ghritlahre, H., \& Prasad, R. (2018). Application of ANN technique to predict the performance of solar collector systems-A review. Renewable and Sustainable Energy Reviews, 84, 75-88.

32. Saloux, E., \& Candanedo, J. (2019). Modelling stratified thermal energy storage tanks using an advanced flowrate distribution of the received flow. Applied Energy, 241, 34-45.
33. Zhou, B., Xu, D., \&Wu, Q. (2020). Integrated modelling and enhanced utilization of power-to-ammonia for high renewable penetrated multienergy systems. IEEE Transactions on Power Systems, 35(6), 4769-4780.

\section{Submit your manuscript to a SpringerOpen ${ }^{\circ}$ journal and benefit from:}

- Convenient online submission

- Rigorous peer review

Open access: articles freely available online

- High visibility within the field

- Retaining the copyright to your article

Submit your next manuscript at $\mathbf{s p r i n g e r o p e n . c o m ~}$ 